\title{
Implementing and evaluating a set of online virtual experimental scenarios for teaching developmental biology
}

\section{Daniel Levin}

University of Manchester, UK

\section{Keith Brennan}

University of Manchester, UK

\section{Adam Hurlstone \\ University of Manchester, UK}

\section{Kathryn E. Hentges}

University of Manchester, UK

\section{Abstract}

Teaching developmental biology to undergraduates can be complicated because it is a discipline most undergraduate students have never experienced before their first in-depth developmental biology course. This case study presents a set of online scenarios created to aid our students in learning developmental biology concepts, in which the student assumes the role of a postgraduate research student. These scenarios complement the course unit Principles of Developmental Biology taught to second-year undergraduate students. In each scenario students must work through experimental simulations and predict their results, answering a series of questions related to concepts taught in accompanying lectures or interpretations of data presented in the scenario. The scenarios focus on concepts common to developmental processes in animals. Additionally, one scenario is solely based on plant development. Student evaluations of these scenarios are very positive. Additionally, students report that working through the scenarios improves their knowledge of experimental techniques. Extending student knowledge of experimental protocols and data interpretation through eLearning approaches is an important addition to the course unit, as the unit is not supplemented by any specific laboratory work.

Keywords: developmental biology; scenario; scenario-based learning; virtual experiments; eLearning. 


\section{Background}

In biology education, learning can be facilitated by incorporating experimental work into lecture-based courses, which gives students an opportunity to test concepts presented in lectures in a hands-on fashion. It is often through this practical application of basic principles that students achieve a true understanding of the lecture material. The use of practical approaches can be especially useful for disciplines that are introduced primarily at the undergraduate level, such as developmental biology. Indeed, prior assessments of student engagement with developmental biology laboratory exercises has shown that students who find laboratory exercises interesting also report plans to take additional courses in the subject area (D'Costa and Shepherd, 2009). However, adding experimental sections to a course has limitations in terms of financial resources, time, and safety considerations. For these reasons, we created a series of virtual laboratory experiments to accompany a second-year undergraduate course entitled 'Principles of Developmental Biology'. These scenarios are accessed by students online, and allow students to play the role of a graduate level researcher performing developmental biology experiments.

While there are web resources for visualising embryos during development (Nieder and Nagy, 2002; Silva-Lopes and Monteiro-Leal, 2003; Hotta et al., 2007; Marsh et al., 2008; Yamada et al., 2010), many of these resources are designed to allow an inspection of anatomical structures throughout gestation. Our online scenarios differ from other resources in that they incorporate role-play to allow mock experiments to be performed by the user. In a manner similar to Problem-Based Learning, these scenarios engage students in active learning that supplements lecture-based delivery of course material. The diversification of content delivery, especially incorporating active learning approaches, has been shown to be beneficial for the teaching of developmental biology (Knight and Wood, 2005; Douglas 2008). Rather than basing our scenarios on specific model organisms, we have invented a new model organism, the Chocolate Monster. The use of a novel organism requires students to apply concepts from lectures to new situations, and also allows a variety of experimental techniques to be simulated in the virtual model organism without the limitations posed by biological differences in actual model systems.

After running these scenarios alongside lectures for a period of 6 years, we sought to evaluate student response to the scenarios. Every year we examined student assessment of the utility and learning benefits delivered from the scenarios using a Lickert scale for set 
questions and free text responses on a paper survey. We found that overall students' responses to the scenarios have been extremely positive, with the majority of students reporting an improved understanding of experimental approaches used in developmental biology research.

\section{Materials used}

\section{Scenario creation}

Initially, concepts amenable to virtual experimentation were identified. A storyline for each scenario was developed and written to include quiz questions. Feedback for incorrect answers on each question was also written to help students work their way through the scenario correctly. Images needed for the scenario were constructed in Powerpoint or Paint. Additionally, photos of laboratory equipment, as well as general laboratory and office locations, were taken. The scenarios were assembled using Scenario Builder Interactive (SBLi) software (Jinks et al., 2011) and hosted locally using SBLi server software, which allowed student progress to be tracked.

\section{Scenario delivery}

Three lecturers teaching the Principles of Developmental Biology course $(\mathrm{KB}, \mathrm{AH}$, and $\mathrm{KEH}$ ) worked alongside an eLearning technologist (DL) to create a series of five scenarios for students to access during the Principles of Developmental Biology course unit. A new scenario was available every two weeks throughout the semester and all scenarios were made available again at the end of the course for students to review prior to exams. The scenario interface has four windows (Figure 1) which show possible locations (top left), actions and collections (bottom left), the current location (top right), and text associated with the current location (bottom right). Students were directed to read the initial text describing the premise of the scenario. Students then chose between various actions or locations to progress throughout the scenario. We introduced dead end points when the wrong experiment or incorrect interpretation of data was chosen. Although we incorporated a limited degree of branching within the scenarios, complex branching was difficult to achieve using the SBLi software. Instead we relied on incorporating end points that sent the students back to an earlier point in the scenario so that they could choose alternative experiments to return to the correct path through the scenario. We predicted each scenario 
would take approximately 1.5 hours for students to complete. Scenarios were accessed through the virtual learning environment Blackboard and hosted on our in house server.

Figure 1. The scenario interface. This shows part of scenario 1 where students must collect the reagents required to synthesise a probe for an in situ hybridisation experiment. The top left panel shows locations available, the top right panel shows the current location, the bottom left panel shows items the student has collected (eggs for analysis), and the bottom right panel shows the text associated with the location (instructions to collect reagents to generate an in situ hybridisation probe). To progress from this stage of the scenario students must collect the reagents they require for generating an in situ hybridisation probe by choosing one of the highlighted items in the location panel and dragging it into the lower right corner of the panel.

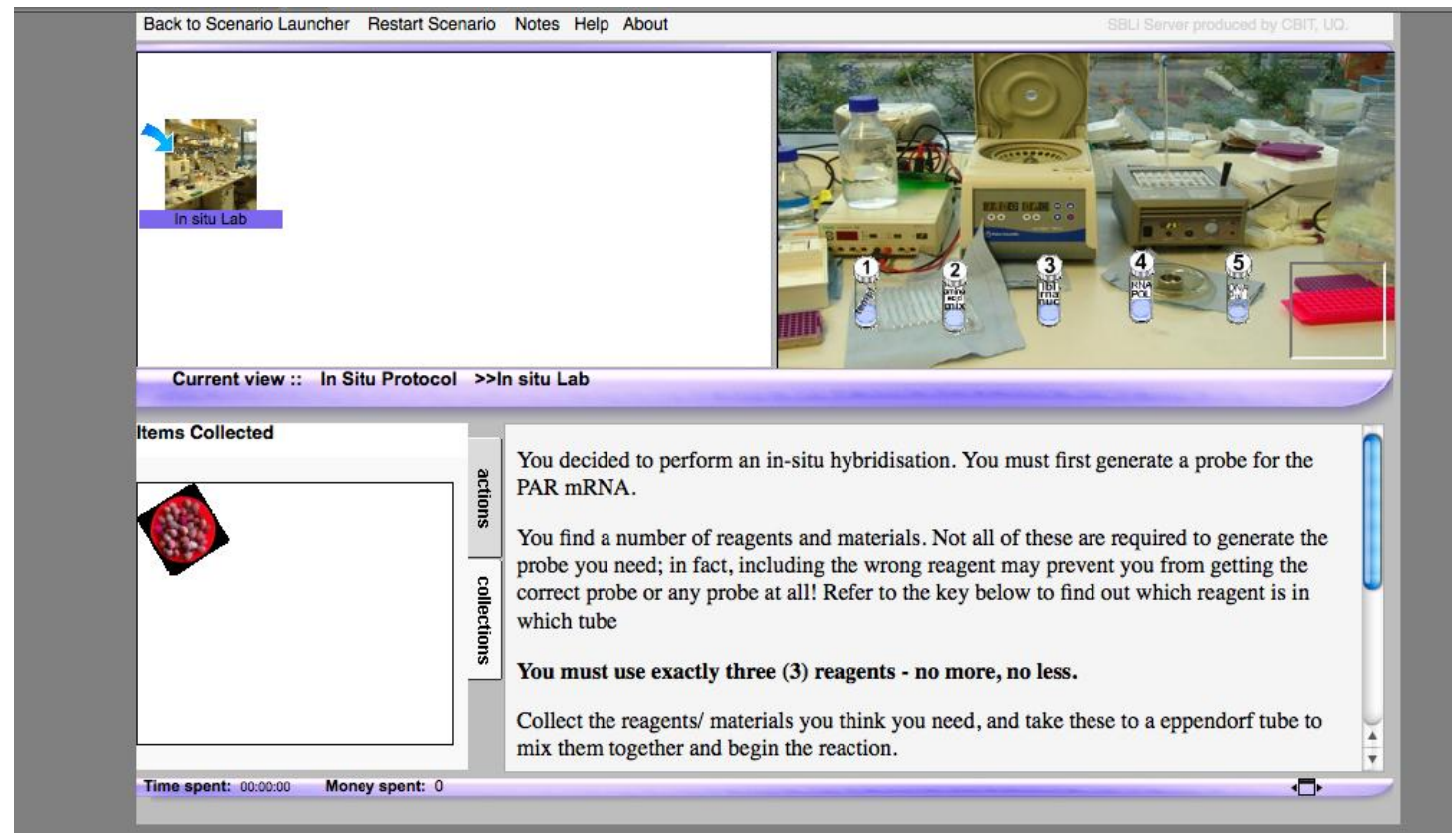

The scenarios had quiz questions incorporated within the scenarios (Figure 2). Students received feedback on the quiz questions and needed to answer each question correctly to continue the scenario. Students were able to attempt each quiz question multiple times until a correct answer was achieved. After the end of new lecture material, students took an additional in-class scenario under exam conditions. This exam scenario differed from the prior scenarios in that each question was marked and students received a percentage score based on the number of questions answers correctly during the scenario. Students did not receive feedback on their answer choices and did not need to enter the correct answer to progress through the exam scenario. 
Figure 2. The use of quiz questions within the scenario. Students answer questions shown in the bottom right panel, based on experimental results given in the scenario in the top right panel.

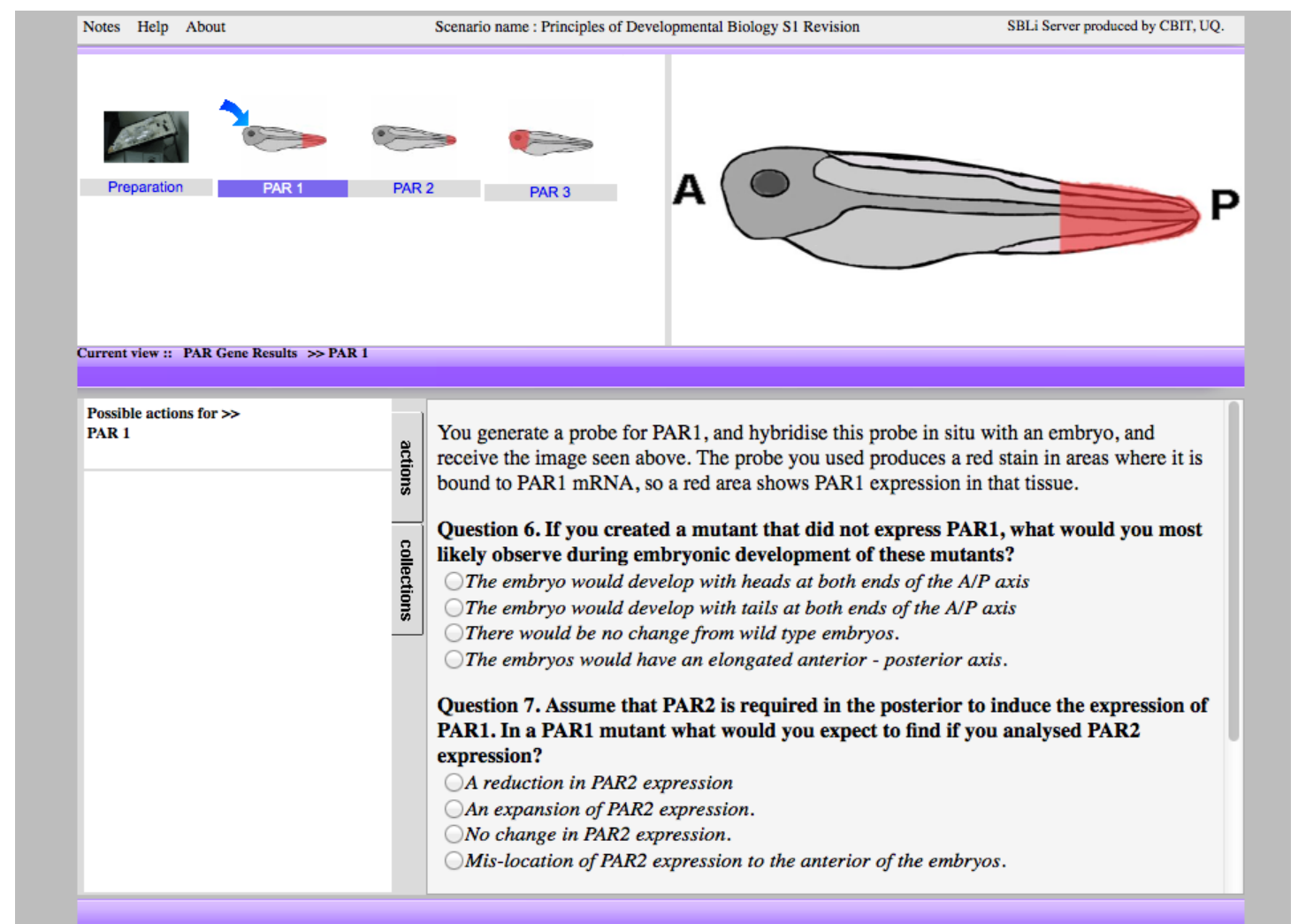

\section{Scenario evaluation}

Students were asked to complete a survey on the scenarios after completing scenario 5 .

There were no marks associated with completing the survey and no penalties for failing to complete it. We collected anonymous data from students taking the unit over a 6-year period and analysed these data to determine students' impressions of the scenarios. This study was approved by the Faculty of Life Sciences ethics review committee; the committee determined that full ethics approval through the University Research Ethics Committee was not necessary for this study, given that the data were collected anonymously. The study followed general good practice as set out by the university for the conduct of student surveys.

Using a Lickert scale students ranked their agreement with the following statements:

- Working through the scenarios improved my understanding of the subject area. 
- Working through the scenarios required me to apply concepts taught in lectures to new situations.

- The content of the scenarios was interesting.

- The content of the scenarios was aimed at an appropriate level.

- I found the scenarios easy to navigate.

Students were also asked to give the set of scenarios an overall rating of excellent, good, average, poor or very poor. Students overwhelmingly gave the scenarios positive ratings (Table 1).

Table 1. Student evaluation of scenarios accompanying Principles of Developmental Biology. Student response to scenario survey.

\begin{tabular}{|l|l|l|l|}
\hline YEAR & $\begin{array}{l}\text { Total } \\
\text { responses }\end{array}$ & $\begin{array}{l}\text { \% rating } \\
\text { scenarios good } \\
\text { or excellent }\end{array}$ & $\begin{array}{l}\text { \% rating } \\
\text { scenario poor } \\
\text { or very poor }\end{array}$ \\
\hline $2008 / 09$ & 50 & 88 & 4 \\
\hline $2009 / 10$ & 93 & 87 & 5 \\
\hline $2010 / 11$ & 87 & 82 & 7 \\
\hline $2011 / 12$ & 148 & 89 & 3 \\
\hline $2012 / 13$ & 152 & 88 & 2 \\
\hline $2013 / 14$ & 95 & 89 & 3 \\
\hline
\end{tabular}

In addition to responding to survey questions students were asked to enter any free text comments they had on the scenarios. In years 2008/09 and 2009/10, these comments were collected in the online survey after the completion of the final scenario. From 2010/11 onwards, free text comments were collected on a paper survey used for the evaluation of the entire course. Some of the positive comments included phrases such as:

The fact that they were interactive really helped.

I really enjoyed these [scenarios] and they did further my knowledge of the lecture material.

Complemented the course unit very well. Wished that they had these for all course units!

I thoroughly enjoyed these scenarios. 
The feedback when an answer was wrong was really useful.

Generally an excellent accompaniment to lectures.

I think the scenarios provide a good study aid helping pool information learnt in lectures.

Most negative comments on the student evaluation forms have focused on technical aspects of the scenario builder interface, such as the difficulty of collecting and uncollecting objects in different locations in the scenarios. Some students felt the interface was simplistic and clunky. Each year a few students commented that they would have rather just had a standard quiz rather than a story to follow in the scenarios. However, the majority of students find the online scenarios a valuable addition to the course unit. Overall, $81 \%$ of student free text comments contain positive statements about the addition of the scenarios to the course.

\section{Implications}

Getting students to engage with course material is a priority within university level education (Harper and Quaye, 2009). In science subjects in particular, students can feel that lecture material alone is insufficient to allow them a deep understanding of the subject matter. The incorporation of new educational tools that students can access in their own time outside of lectures provides a diversification of content delivery that appeals to students with a variety of learning styles, including kinaesthetic learning (Fleming, 2001). A recent study suggests that modern students prefer bodily-kinaesthetic learning styles, concluding that the incorporation of digital storytelling will motivate and engage current students (Sandars and Homer, 2008).

Although students' responses to the scenarios have been overwhelmingly positive, there are some drawbacks associated with the use of scripted scenarios in teaching developmental biology. We have designed the scenarios to require students to make choices about the experiments they would perform and the reagents they would require. However, the scenarios cannot account for every independent choice students would make on their own. Also, the degree of branching within the scenarios is limited, so that 
students are sent back to a specific point in the scenarios following an incorrect choice. Allowing students to choose a different approach at a dead end point does not completely accurately replicate an experimental setting where using incorrect reagents or the wrong technique would prevent the student from obtaining meaningful results.

The quiz questions in each scenario were designed to examine the application of concepts by asking students to interpret mock data generated in virtual experiments. The quiz questions also informed students of gaps in their own knowledge by providing feedback for incorrect answers. Teaching developmental biology through the application of concepts rather than factual recall has been encouraged (Wood, 2008), and we have attempted to allow students to actively apply developmental biology concepts within virtual experiments, which students report has improved their understanding of the topics introduced in lectures. Although we have developed these scenarios for teaching developmental biology, similar scenarios could be developed for a variety of subjects to facilitate active learning and application of conceptual information.

\section{Accessing materials}

The scenarios described in this article can be accessed at:

http://sbli.Is.manchester.ac.uk/Demo (username: 'developmental' password: 'biology').

\section{Acknowledgements}

We would like to thank Geoff Norton and the SBLi team for helpful suggestions on using SBLi Builder software. We thank the University of Manchester Principles of Developmental Biology students for their feedback on using the scenarios. We thank Dr. M. Danilchik, Oregon Health Sciences University, for providing Xenopus images for use in scenarios. 


\section{References}

D'Costa, A. and Shepherd I.T. (2009) 'Zebrafish development and genetics: introducing undergraduates to developmental biology and genetics in a large introductory laboratory class', Zebrafish, 6(2), pp. 169-177.

Douglas, K.R. (2008) 'A kinesthetic model demonstrating molecular interactions involved in anterior-posterior pattern formation in Drosophila', CBE Life Sciences Education, 7(1), pp. 74-81.

Fleming, N.D. (2001) Teaching and learning styles: VARK Strategies. Christchurch, New Zealand: N.D. Fleming.

Harper, S.R. and Quaye, S. (2009) 'Beyond sameness with engagement and outcomes for all', in Harper, S.R. and Quaye, S. (eds.) Student engagement in higher education. Abingdon: Routledge, pp. 1-16.

Hotta, K., Mitsuhara, K., Takahashi, H., Inaba, K., Oka, K., Gojobori, T. and Ikeo, K. (2007) A web-based interactive developmental table for the ascidian Ciona intestinalis, including 3D real-image embryo reconstructions: I. From fertilized egg to hatching larva', Developmental Dynamics, 236(7), pp. 1790-1805.

Jinks, A., Norton, G., Taylor, M. and Stewart, T. (2011) ‘Scenario-based learning: experiences in the development and application of a generic teaching software tool', in Holt, D., Segrave, S. and Cybulski, J. (eds.) Professional education using esimulations: benefits of blended learning design. USA: IGI Global, pp. 346-369.

Knight, J.K. and Wood, W.B. (2005) 'Teaching more by lecturing less', Cell Biology Education, 4(4), pp. 298-310.

Marsh, K.R., Giffin, B.F. and Lowrie D.J. Jr. (2008) 'Medical student retention of embryonic development: impact of the dimensions added by multimedia tutorials', Anatomical Science Education, 1(6), pp. 252-257. 
Nieder G.L. and Nagy F. (2002) 'Analysis of medical students' use of web-based resources for a gross anatomy and embryology course', Clinical Anatomy, 15(6), pp. 409-418.

Sandars J. and Homer M. (2008) 'Reflective learning and the Net Generation', Med Teach. 30(9-10), pp. 877-879.

Silva-Lopes V.W. and Monteiro-Leal L.H. (2003) 'Creating a histology-embryology free digital image database using high-end microscopy and computer techniques for online biomedical education', The Anatomical Record Part B: The New Anatomist, 273B(1), pp. 126-131.

Wood, W.B. (2008) 'Teaching concepts versus facts in developmental biology', CBE Life Science Education, 7(1), pp. 10-11.

Yamada, S., Samtani, R.R., Lee, E.S., Lockett, E., Uwabe, C., Shiota, K., Anderson, S.A. and Lo, C.W. (2010) 'Developmental atlas of the early first trimester human embryo', Developmental Dynamics, 239(6), pp. 1585-1595.

\section{Author details}

Daniel Levin is an eLearning Technologist at the University of Manchester. He holds a BSc in Genetics from the University of Manchester.

Keith Brennan is a Professor of Developmental Biology at the University of Manchester and a Deputy Associate Dean for Education.

Adam Hurlstone is a Senior Lecturer at the University of Manchester.

Kathryn E. Hentges is a Senior Lecturer at the University of Manchester. 